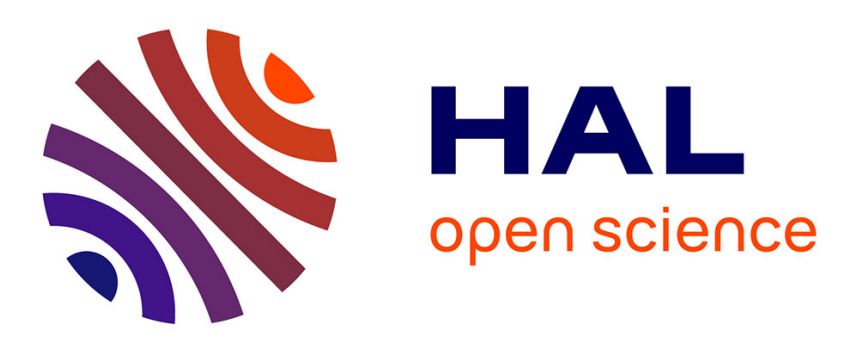

\title{
Sensitivity to central crowding for faces in patients with glaucoma
}

\author{
Aymeric Stievenard, Jean Francois Rouland, Carole Peyrin, Aude Warniez, \\ Muriel Boucart
}

\section{> To cite this version:}

Aymeric Stievenard, Jean Francois Rouland, Carole Peyrin, Aude Warniez, Muriel Boucart. Sensitivity to central crowding for faces in patients with glaucoma. Journal of Glaucoma, 2021, 30 (2), pp.140-147. 10.1097/IJG.0000000000001710 . hal-03017651

\section{HAL Id: hal-03017651 https://hal.science/hal-03017651}

Submitted on 21 Nov 2020

HAL is a multi-disciplinary open access archive for the deposit and dissemination of scientific research documents, whether they are published or not. The documents may come from teaching and research institutions in France or abroad, or from public or private research centers.
L'archive ouverte pluridisciplinaire HAL, est destinée au dépôt et à la diffusion de documents scientifiques de niveau recherche, publiés ou non, émanant des établissements d'enseignement et de recherche français ou étrangers, des laboratoires publics ou privés. 


\section{Journal of Glaucoma \\ Sensitivity to central crowding for faces in patients with glaucoma \\ --Manuscript Draft--}

\begin{tabular}{|c|c|}
\hline \multicolumn{2}{|l|}{ Manuscript Number: } \\
\hline Full Title: & Sensitivity to central crowding for faces in patients with glaucoma \\
\hline Article Type: & Original Study \\
\hline Keywords: & glaucoma, face perception, crowding, masking \\
\hline Corresponding Author: & $\begin{array}{l}\text { Muriel Boucart, PhD } \\
\text { Centre National de la Recherche Scientifique } \\
\text { lille, nord FRANCE }\end{array}$ \\
\hline \multicolumn{2}{|l|}{$\begin{array}{l}\text { Corresponding Author Secondary } \\
\text { Information: }\end{array}$} \\
\hline Corresponding Author's Institution: & Centre National de la Recherche Scientifique \\
\hline \multicolumn{2}{|l|}{$\begin{array}{l}\text { Corresponding Author's Secondary } \\
\text { Institution: }\end{array}$} \\
\hline First Author: & Aymeric Stievenard, MD \\
\hline \multicolumn{2}{|l|}{ First Author Secondary Information: } \\
\hline \multirow[t]{5}{*}{ Order of Authors: } & Aymeric Stievenard, MD \\
\hline & Jean Francois Rouland, MD PhD \\
\hline & Carole Peyrin, PhD \\
\hline & Aude Warniez, MSc \\
\hline & Muriel Boucart, PhD \\
\hline \multicolumn{2}{|c|}{ Order of Authors Secondary Information: } \\
\hline Manuscript Region of Origin: & FRANCE \\
\hline Abstract: & $\begin{array}{l}\text { Purpose: to investigate whether face recognition difficulties reported by some patients } \\
\text { with glaucoma result from a greater sensitivity to inner crowding in central vision. } \\
\text { Method: Seventeen patients with glaucoma and } 17 \text { age-matched normally sighted } \\
\text { controls participated. An isolated mouth (uncrowded condition) or a mouth within a face } \\
\text { (crowded condition) was randomly displayed centrally for } 200 \text { ms. For each condition, } \\
\text { participants were asked to decide whether the mouth was closed or open. The stimuli } \\
\text { were presented at three angular sizes }\left(0.6^{\circ} \times 0.4^{\circ}, 1^{\circ} \times 0.72^{\circ} \text {, and } 1.5^{\circ} \times 1.08^{\circ}\right) \text {. } \\
\text { Accuracy was measured. } \\
\text { Results: Crowding affected performance differentially for patients and controls. } \\
\text { Consistent with previous studies controls exhibited a "face superiority effect", with a } \\
\text { better accuracy when the mouth was located within the face than when it was isolated } \\
\text { A sensitivity to crowding, reflected in a better accuracy with the isolated mouth, was } \\
\text { observed in } 10 \text { out of } 17 \text { patients only for small images. Crowding disappeared for } \\
\text { larger faces, as the facial features were spaced out. Five patients were not sensitive to } \\
\text { crowding. Importantly, no difference was found between the two subgroups of patients } \\
\text { (sensitive vs non sensitive) in terms of Mean Deviation, contrast sensitivity, acuity, } \\
\text { thickness of the RNLF or macular GCIPL. } \\
\text { Conclusion: An excessive sensitivity to central crowding might explain the difficulties in } \\
\text { face perception and reading reported by some patients with glaucoma. The sensory or } \\
\text { cognitive processes underlying this excessive sensitivity must be elucidated to improve } \\
\text { central perception in glaucoma. }\end{array}$ \\
\hline
\end{tabular}


The photo number 18 of the NimStim database is allowed for publication in scientific journals. "Only models $\# 01,03,18,28,40$, \& 45 may be published, and these may only be published in scientific journals. Researchers may not publish images from the remaining models anywhere."

(https://www.macbrain.org/resources.htm).

best regards

Muriel Boucart 
Lille, 10 June 2020

Dear Pr Liebmann,

Please find enclosed a research article entitled "Sensitivity to central crowding for faces in patients with glaucoma" by A Stievenard, JF Rouland, C Peyrin, A Warniez \& M Boucart that we would like to submit for publication in $\mathrm{J}$ of Glaucoma.

It is a psychophysical study on face perception in patients with glaucoma. We test the hypothesis of a higher sensitivity to central crowding in glaucoma that would explain the difficulties reported by some patients to recognize faces seen at a distance of about $18-20 \mathrm{~m}$. The results are consistent with Ogata et al. (2019) who found a higher sensitivity to crowding in glaucoma compared to controls. However, they tested crowding in peripheral vision at $10^{\circ}$ where crowding is usually found in normally sighted people. We show that the higher sensitivity to crowding extends to central vision in some patients (10/17) likely due to a reduced contrast sensitivity. In crowding the presence of nearby flankers (here the other facial features) reduces the contrast of the target (here the mouth). The results of the normally sighted controls are consistent with the literature.

The English of the manuscript has been checked by a native English speaker.

Best regards

Muriel Boucart 
Aymeric Stievenard $^{1}$ (MD), Jean François Rouland (MD-PhD) $)^{1-2}$, Carole Peyrin $\mathrm{PhD})^{3}$ Aude Warniez $(\mathrm{MSc})^{2} \&$ Muriel Boucart $(\mathrm{PhD})^{2}$

\section{Sensitivity to central crowding for faces in patients with glaucoma}

1. Centre Hospitalier Universitaire de Lille, Hôpital Huriez, Service d'Ophtalmologie, Lille, France

2. Univ. Lille, Inserm, CHU Lille, UMR-S 1172 - Lille Neurosciences \& Cognition, 59000 Lille, France

3. Univ. Grenoble Alpes, Univ Savoie Mont Blanc, CNRS, LNPC, 38000 Grenoble, France

Corresponding author: Muriel Boucart, Faculté de Médecine de Lille, Pôle

Recherche, 1 place de Verdun, 59000 Lille, France. E-mail: muriel.boucart@chrulille.fr

Running head: Central crowding in glaucoma

\section{Financial support: none}

Disclosure: none for all of the authors 
Some patients with glaucoma report difficulties to recognize faces when they are far away.

We show that this deficit could result from a higher sensitivity to crowding in central vision.

\begin{abstract}
Purpose: to investigate whether face recognition difficulties reported by some patients with glaucoma result from a greater sensitivity to inner crowding in central vision.

Method: Seventeen patients with glaucoma and 17 age-matched normally sighted controls participated. An isolated mouth (uncrowded condition) or a mouth within a face (crowded condition) was randomly displayed centrally for $200 \mathrm{~ms}$. For each condition, participants were asked to decide whether the mouth was closed or open. The stimuli were presented at three angular sizes $\left(0.6^{\circ} \times 0.4^{\circ}, 1^{\circ} \times 0.72^{\circ}\right.$, and $\left.1.5^{\circ} \times 1.08^{\circ}\right)$. Accuracy was measured.
\end{abstract}

Results: Crowding affected performance differentially for patients and controls. Consistent with previous studies controls exhibited a "face superiority effect", with a better accuracy when the mouth was located within the face than when it was isolated. A sensitivity to crowding, reflected in a better accuracy with the isolated mouth, was observed in 10 out of 17 patients only for small images. Crowding disappeared for larger faces, as the facial features were spaced out. Five patients were not sensitive to crowding. Importantly, no difference was found between the two subgroups of patients (sensitive vs non sensitive) in terms of Mean Deviation, contrast sensitivity, acuity, thickness of the RNLF or macular GCIPL.

Conclusion: An excessive sensitivity to central crowding might explain the difficulties in face perception and reading reported by some patients with glaucoma. The sensory or cognitive processes underlying this excessive sensitivity must be elucidated to improve central perception in glaucoma.

\title{
Key words: glaucoma, face perception, crowding, masking
}




\section{Introduction}

Glaucoma is an ocular pathology characterized by progressive loss of retinal ganglion cells and optic nerve fibers. It is classically associated with peripheral visual field defect, sparing central vision. However, growing evidence indicates physiological impairments in central vision, even at an early stage,$^{1-2}$ with an impact on behavioral functions relying on central vision, such as reading ${ }^{3-4}$ and face recognition. Although face recognition difficulties are not a major complaint among glaucoma patients, several studies have reported impairments, either self-reported in questionnaires ${ }^{5}$ or in psychophysical experiments. ${ }^{6-9}$ For instance, with the Cambridge Face Memory Test in which participants have to recognize previously seen faces within new faces in forced-choice recognition trials, Glen et al. ${ }^{6}$ showed that people with advanced glaucomatous visual field defects, specifically within the central $10^{\circ}$, performed worse at face recognition than people with less advanced defects and age-matched controls. With the same face memory test, Mazzoli et al. ${ }^{9}$ also reported that patients at a severe stage of glaucoma had lower face recognition performance. The mechanisms underlying the deficit in face recognition have not yet been elucidated. Glen et al. ${ }^{10}$ suggested that the deficit might partly result from impaired visual exploration in people with glaucoma. Roux-Sibilon et al. ${ }^{7}$ showed that glaucomatous patients with a central visual field defect were impaired in the categorization of centrally displayed faces at low contrast (2.5\%), suggesting a deficit in contrast sensitivity as a difficulty factor in face perception. In that same study, increasing contrast from $2.5 \%$ to $10 \%$ of the same stimuli improved face categorization in patients. Schafer et al. ${ }^{8}$ measured the distance threshold for gender recognition and the facial expression of centrally displayed colored faces. The faces were first presented at an angular size, simulating faces viewed from 20 meters. The size increased progressively, simulating a person approaching. A key press stopped the size increase. They showed that patients with glaucoma required a larger size (a shorter distance) to recognize both the gender and the facial expression. The authors suggested that a greater sensitivity to crowding in central vision might be responsible for the need for larger faces in patients with glaucoma.

Visual crowding is defined as a difficulty in identifying a target in the presence of nearby flankers. Crowding has been demonstrated extensively with various visual stimuli such as verniers, letters, objects, faces, and scenes. ${ }^{11}$ Several accounts have been proposed to explain this robust phenomenon. For complex stimuli like words, letter-like symbols or faces, crowding is thought to result from an inappropriate feature combination producing changes in 
appearance (a scrambled perception), as demonstrated by asking observers to draw crowded stimuli. ${ }^{12}$ Stimuli like words and faces are composed of elements (letters, facial features) that crowd each other (inner crowding) ${ }^{13}$ when the target-flanker distance is smaller than 0.5 times the eccentricity of the target (Bouma's law). ${ }^{14}$ In normally sighted observers, crowding is particularly strong in peripheral vision, where visual sensitivity is reduced. ${ }^{15} \mathrm{In}$ peripheral vision, targets that could easily be recognized in isolation are unrecognizable when surrounded by close-by elements. As peripheral vision is impaired in glaucoma, crowding is even stronger in patients. This was demonstrated by Ogata et al. ${ }^{16}$, who found that the critical spacing between a central letter and two adjacent flanker letters had to be larger than that of normally sighted controls for patients to escape crowding at $10^{\circ}$ eccentricity.

As patients with glaucoma may also exhibit reduced sensitivity in central vision ${ }^{6-7}$ and several studies have reported impaired face recognition, the present study was undertaken to assess whether the difficulties encountered by some patients in recognizing faces ${ }^{5}$ result from greater sensitivity to crowding in central vision. We compared performance for an isolated facial feature (a mouth) and for the same facial feature in its appropriate location within a face. In both conditions (face/isolated), participants were asked to discriminate the state of the mouth: open or closed. We also manipulated the angular size of the stimuli. We hypothesized that accuracy would be higher for the isolated mouth (uncrowded condition) than for the mouth within a face (crowded condition) for patients but not for controls, as crowding is observed in peripheral vision for which sensitivity is reduced in normally sighted people. Foveal vision is not immune to crowding in normally sighted people, but it is observed for tiny optotypes or verniers with critical spacings lower than $2-5$ arcminute. ${ }^{17-18} \mathrm{We}$ expected an interaction between crowding and size in patients. We hypothesized that increasing the angular size would eliminate crowding (i.e., the better performance for the isolated mouth than for the mouth within a face), since enlarging the whole face increases the spacing between elements and thus reduces inner crowding. Finally, Ogata et al. ${ }^{16}$ measured sensitivity to crowding in glaucoma at $10^{\circ}$ eccentricity. They found that the greater sensitivity to crowding in patients with glaucoma was significantly associated with retinal nerve fiber layer thickness (RNFL) measurements, but not with standard automated perimetry. We tested associations between performance and RNFL, macular Ganglion Cell-Inner Plexiform Layer (GCIPL), mean deviation at the 10-2 visual field test, acuity, and contrast sensitivity. 


\section{METHOD}

120

$\underline{\text { Participants }}$

The characteristics of the two populations are summarized in Table 1. Seventeen patients (10 females) with visual field (VF) defects in both eyes due to primary open-angle glaucoma were asked to participate. The patients ranged from 24 to 72 years of age (mean $58.3 \pm 10$ ). Each patient underwent a complete ophthalmological examination including a visual field evaluation just before the experiment. Visual field sensitivity (expressed as the mean deviation: MD) was measured with a 10-2 Humphrey Field Analyzer (HFA, Carl Zeiss Medical, CA, USA). The 10-2 visual field test measures 68 spotlights in the central $10^{\circ}$ of the visual field. We also measured binocular visual acuity, binocular contrast sensitivity using the Pelli Robson chart and OCT (Optical Coherence Tomography) to assess the local thickness of the RNFL (Retinal Nerve Fiber Layer) and the macular GCIPL (Ganglion Cell-Inner Plexiform Layer). The 10-2 visual fields of patients are displayed in Figure 1.

Seventeen age-matched controls ( 8 females), ranging from 28 to 73 years in age (mean: $57.9 \pm 10$ ), were recruited among the patients' relatives and friends. The inclusion criteria for patients and controls were the following: no history of neurological and/or psychiatric disease, no ocular disease other than glaucoma for patients, and no family history of glaucoma for controls.

For both groups, a binocular acuity lower than $8 / 10$ (Snellen equivalent) and chronic use of drugs that can affect attention (benzodiazepines) were exclusion criteria. The patients and the age-matched controls over 65 were assessed with the French version of the Mini Mental State Examination (MMSE) ${ }^{19}$ to check for cognitive impairment. Participants whose MMSE score was lower than 25/30 were excluded from the study. Age-matched controls received an ophthalmological examination prior to study inclusion to rule out any sign of glaucoma. All participants were asked to come with their habitual optical correction. Older patients and age-matched controls wore progressive spectacles for close and distant vision. When the acuity test performed before the experimental session showed that the participant's optical correction was not appropriate at the viewing distance chosen for the experiment, the ophthalmologist (author AS) provided a suitable correction. Patients and age-matched 
controls did not differ significantly in age $(\mathrm{F}(1,32)=0.008, \mathrm{p}=0.928)$, visual acuity $(\mathrm{F}(1$, $32)=0.314, p=0.579)$ or contrast sensitivity $(F(1,32)=1.36, p=0.251)$. The study was approved by the ethics committee of the University of Lille. In accordance with the tenets of the Declaration of Helsinki, written informed consent was obtained from all participants.

[Table 1 and Figure 1 about here]

Stimuli: The stimuli were colored photographs of 10 male and 10 female faces exhibiting a neutral facial expression, selected from the NimStim sorted emotions database. ${ }^{20}$ For each gender, there were 5 faces with an open mouth and 5 faces with a closed mouth. Each face was presented on a light grey background screen. An "isolated mouth" version was created from each face. For each face, the mouth was extracted using the Gimp 2.8 software and pasted on a light grey rectangle, keeping the same size and spatial location as that of the faces. Examples are shown in Figure 2.

[Figure 2 about here]

Procedure: The software of the experiment was written in Matlab. Participants were seated at a viewing distance of $57 \mathrm{~cm}$ from a 30-inch screen (DELL). The stimuli were presented in photopic conditions with light coming from the ceiling. Recent laboratory evidence has shown that crowding under photopic conditions is stronger than under mesopic luminance. ${ }^{21}$ At the beginning of the experiment, participants were shown an example of 2 faces and 2 mouths (open/closed) on paper. They were told that faces, or isolated mouths, were going to be displayed randomly on the screen and that they would have to decide whether the mouth was open or closed. For each trial, a central black fixation cross was displayed for $1 \mathrm{sec}$ on a grey background. It was followed $100 \mathrm{~ms}$ later by a face or an isolated mouth displayed centrally for $200 \mathrm{~ms}$. Each face was randomly presented in three sizes: $0.6^{\circ} \mathrm{X} 0.4^{\circ}, 1^{\circ} \mathrm{X} 0.72^{\circ}$ and $1.5^{\circ}$ $\mathrm{X} 1.08^{\circ}$. The smallest angular size corresponds to the threshold of patients with glaucoma for the recognition of the gender of a face in Schafer et al. ${ }^{8}$ The mouths were extracted from the faces. Participants were tested in binocular vision. A training session of 20 trials, with different stimuli from those used in the experiment, was performed to familiarize the participants with the exposure duration. It was followed by 120 experimental trials (20 faces + 
20 isolated mouths X 3 angular sizes). Participants responded orally (open/closed mouth). The answer was entered on the keyboard of the computer by the experimenter.

\section{RESULTS}

An ANOVA was conducted on the percentage of correct responses with the Systat 8 (Systat Software, Inc. San Jose, California) software. The group (patients/age-matched controls) was the between-subject factor. The three angular sizes of the images and the crowding (mouth in a face versus isolated mouth) were the within-subject factors.

On average, performance did not differ significantly between patients and controls $(77 \%$ vs $79.4 \%$ of correct responses; $\mathrm{F}(1,32)=0.86, \mathrm{p}=0.36)$, indicating that the task was not more difficult for patients than for controls. The main effect of angular size was significant $(\mathrm{F}(2,64)=149, \mathrm{p}<.001)$. Accuracy increased with the increase in angular size, both for controls $\left(0.6^{\circ}: 66 \%, 1^{\circ}: 82 \%, 1.5^{\circ}: 90 \%\right)$ and for patients $\left(0.6^{\circ}: 66.5 \%, 1^{\circ}: 78.7 \%\right.$, $\left.1.5^{\circ}: 86 \%\right)$. There was no significant interaction between size and group $(\mathrm{F}(2,64)=1.84, \mathrm{p}=$ $0.167)$.

Sensitivity to crowding is reflected in greater accuracy for the isolated mouth than for the mouth in a face. Crowding affected performance differentially for patients and controls, as indicated by a significant interaction between group and stimuli $(\mathrm{F}(1,32)=4.97, \mathrm{p}<.033)$. As can be seen in Figure 3, controls and patients did not differ significantly in the uncrowded (isolated mouth) condition (controls: $75.5 \%$, patients: $75.1 \%, \mathrm{~F}(1,32)=0.13, \mathrm{p}=0.71$ ) but the patients' performance was significantly lower than that of controls in the crowded (face) condition (controls: 83.5\%, patients: $77.5 \%, \mathrm{~F}(1,32)=4.51, \mathrm{p}<.042$ ). Controls exhibited a "face superiority effect" with greater accuracy when the mouth was located in the face than when it was isolated $(83.5 \%$ vs $75.5 \%, \mathrm{~F}(1,16)=14.7, \mathrm{p}<.001)$.

[Figure 3 about here]

On average, accuracy did not differ significantly for the mouth in a face (77.5\%) and the isolated mouth $(75.1 \%)$ for patients $(\mathrm{F}(1,16)=0.10, \mathrm{p}=0.75)$, but stimuli interacted 
significantly with the angular size in the patient group $(\mathrm{F}(2,32)=5.5, \mathrm{p}<.009)$, whilst it did not in the control group $(F(2,32)=0.962, \quad p=0.393)$. Controls exhibited an advantage for the face, as compared to the isolated mouth, for the three angular sizes (by $5 \%$ at $0.6^{\circ}, 10 \%$ at $1^{\circ}$ and $8 \%$ at $1.5^{\circ}$ ). In contrast, patients exhibited greater accuracy (by 5\%) for the isolated mouth than for the mouth in a face at $0.6^{\circ}$, slightly greater accuracy (by $0.5 \%$ ) for the face than for the isolated mouth at $1^{\circ}$ and greater accuracy for the face (by $7 \%$ ) at $1.5^{\circ}$. Individual data (Figure 4 ) show that 10 out of 17 patients exhibited greater sensitivity to crowding, i.e. a better performance for the isolated mouth (by $15 \% \mathrm{t}(9)=9, \mathrm{p}<.001$ ) for small images $\left(0.6^{\circ}\right)$. Two patients exhibited the same performance for the face and the isolated mouth, and 5 patients were not sensitive to crowding. They exhibited a better performance for the mouth in a face. No significant difference was found between the two subgroups of patients (sensitive vs. non sensitive to crowding) in terms of MD of the best eye $F(1,15)=0.327, p=0.576$, of RNFL of the best eye $F(1,15)=1.54, p=0.23)$, GCIPL of the best eye $(F(1,15)=2.67, p=$ $0.123)$, acuity $(F(1,15)=0.56, p=0.46)$, and contrast sensitivity $(F(1,15)=0.08, p=0.78)$.

[Figure 4 about here]

\section{DISCUSSION}

There is growing evidence that glaucoma impairs functions relying on central vision such as reading ${ }^{4,22}$, face recognition ${ }^{6,8}$ and visual search for objects in scenes ${ }^{23}$. Deficits in contrast sensitivity $^{7,24}$ and visual exploration as well as saccadic eye movements ${ }^{10,23,25}$ have been suggested to account partly for this deficit. We assessed whether excessive sensitivity to central crowding might be another underlying mechanism. In contrast to masking, crowded objects can be detected but they appear scrambled and indistinct. In our study, sensitivity to crowding was expected to be reflected in greater accuracy for an isolated element (a mouth) than for the same element embedded in a face. A variant of this experimental paradigm was used by Martelli et $a^{13}$. They investigated sensitivity to peripheral crowding for faces in normally sighted participants. They selected three mouths from different faces (smiling, neutral, and frowning) and presented them either isolated or in the context of a face. In their study, crowding was assessed by increasing the spacing between the facial elements, i.e. by moving every other facial feature away from the target mouth. They measured a critical 
spacing to identify the target mouth at various eccentricities (from 0 to $12^{\circ}$ ) and sizes $\left(0.4^{\circ}\right.$ to $3.2^{\circ}$ ). They observed that all participants exhibited a face superiority effect in central vision $\left(0^{\circ}\right)$. The mouth was recognized more easily when it was presented in the context of a face than in isolation. The opposite was found for peripheral vision. The other facial features hindered the recognition of the mouth owing to inner crowding.

Consistent with Martelli et al. ${ }^{13}$, we observed a face superiority effect in normally sighted participants in central vision. Accuracy was higher when the mouth was in a face than when it was isolated at the three angular sizes, though statistically significant only at 1 and $1.5^{\circ}$. In contrast, 10 out of 17 patients with glaucoma exhibited a better performance for the isolated mouth than for the mouth in a face at the smallest angular size. The advantage for the isolated mouth decreased with larger faces. This was expected as enlarging the face increased the spacing between the mouth and the other facial features, thus reducing crowding. However, as can be seen in the individual data in Figure 3, sensitivity to crowding was still present with larger faces in some patients, suggesting that for them, the spacing between elements needs to be larger to escape crowding. Our results are also consistent with Ogata et al. ${ }^{16}$, who reported greater sensitivity to crowding in patients with glaucoma. However, in their study, crowding was tested in peripheral vision at $10^{\circ}$ eccentricity and they did not measure crowding in central vision. They reported an association between the critical spacing and the RNFL thickness but not with the MD of the 24-2 visual field test. We found no significant difference in terms of MD, RNFL, GCIPL, contrast sensitivity, MD of the 10-2 visual field test and acuity between our subgroup of patients sensitive to crowding (10 patients who exhibited greater accuracy for an isolated mouth at $0.6^{\circ}$ angular size) and our subgroup of patients not sensitive to crowding (5 patients who did not). Patient 11 and Patient 12, whose performance was similar to that of the controls (face superiority effect), had early stage glaucoma and severe glaucoma, respectively. Patients 13, 14 and 16, who exhibited a strong sensitivity to crowding, had no central visual field deficit.The cause of crowding is still under debate. Various underlying mechanisms have been proposed from sensory (lateral inhibition, spatial pooling) to cognitive processes (attention, contextual modulation, scrambled representation) $)^{11,12,26}$. Ogata et al. ${ }^{16}$ explained the greater sensitivity to peripheral crowding in patients with glaucoma in terms of enlarged areas of receptive field integration due to neural loss. Excessive sensitivity to crowding for patients with letters in peripheral vision, as in Ogata et al. ${ }^{16}$, and to inner crowding with faces in central vision in our experiment might have involved different mechanisms. 
The patients' performance for small faces could reflect a greater sensitivity to lateral masking of the mouth by the other facial features. It is difficult to distinguish lateral masking from crowding. For some authors ${ }^{15}$, masking and crowding involve different mechanisms. Masking affects feature detection while crowding impairs feature integration ${ }^{13}$. Crowding is sensitive to the spatial spacing between elements up to a critical spacing (Bouma's law). On the other hand, masking is not sensitive to spacing. Crowding occurs in peripheral vision where sensitivity is reduced, while masking occurs in peripheral and central vision. For others $^{27}$, crowding and lateral masking "are two sides of the same coin" and are considered to share a common mechanism, which is a reduction in contrast sensitivity. Levi et al. ${ }^{27}$ measured the contrast threshold for the discrimination of the orientation of a gabor patch in isolation or flanked by similar patterns. In each trial, the target was presented at one of four near-threshold contrast levels. The observers were asked to rate the magnitude of the contrast (from 0 to 3). They found that, for high contrast flankers, contrast threshold elevation for crowding was similar to contrast threshold elevation usually observed for masking. In the present experiment, it could be that the other facial features induced a reduction in contrast sensitivity for the target mouth. With gabor patches modulated in spatial frequency and contrast, Lahav et al. ${ }^{28}$ found that foveal contrast sensitivity is impaired in glaucoma patients despite good visual acuity, suggesting central visual function damage in glaucoma. Reduced contrast sensitivity has also been reported in apparently intact areas of the visual field in patients with glaucoma and in preperimetric patients diagnosed with $\mathrm{OCT}^{29}$. We found no difference in contrast sensitivity for patients who exhibited crowding and patients who exhibited a face superiority, but the Pelli Robson chart might not be sensitive enough and we did not manipulate contrast.

Other factors, such as fixation stability and reduced attentional processes, might have played a role in the sensitivity to central crowding in some patients. While we did not measure the stability of fixation, Montesano et al. ${ }^{30}$ retrospectively analyzed eye-movement data, especially fixation stability, in 120 patients with glaucoma and 200 normally sighted controls. They used data obtained during the preferred retinal locus registration performed prior to automated perimetry assessment in fundus perimetry. They reported no difference between patients and controls in the Bivariate Contour Ellipse Area (BCEA), a measure of spatial dispersion of fixation while participants fixate a central spot for several seconds (Crossland et al., 2004). However, the Sequential Euclidean Distance (SED), a measure of the temporal instability of fixation, was significantly greater in patients than in controls. The SED is a 
measure of how frequently the participant changes fixation location, independently of the spatial spread of the points. Some patients may have more unstable fixation than others.

In normally sighted observers, covert spatial attention is known to sharpen signal perception in difficult visual conditions, such as low contrast ${ }^{31}$ or crowding ${ }^{32}$. For instance, Yeshurun and Rashal ${ }^{32}$ showed that orienting attention towards the target via a precue reduces the critical spacing between target and flankers to avoid crowding and enhances target identification accuracy. We are not aware of psychophysical studies on covert spatial attention using a cueing paradigm in glaucoma. However, a deficit in covert spatial attention is unlikely in the present study as there was no uncertainty on the spatial location of the stimuli since they were always displayed centrally. Nevertheless, some patients may have focused their attention on the lower part of the face. In the same line, contextual modulation could also be tested to assess whether patients sensitive to crowding analyze faces differently from patients who are insensitive to it, i.e. featural versus global processing. A global processing of the face would interfere with the selection of the mouth. In a study on crowding, Sayim et al. ${ }^{18}$ showed that flankers deteriorate performance on a target when flankers and target form one perceptual group. When target and flankers are not part of the same group (they may differ in color, in contrast polarity ${ }^{33}$ or the flankers are grouped into a figure ${ }^{18}$ ), the target stands out and crowding is reduced or eliminated. This suggests that, if the face interferes with the detection of a target element, e.g. the mouth, then crowding should be reduced if the mouth is embedded in a scrambled face, i.e. when the facial features are not grouped into a facial configuration.

Conclusion and limitations: The findings of the present study confirmed our prediction that one of the underlying mechanisms of impaired face perception in glaucoma is a greater sensitivity to central crowding. However, this conclusion has limitations and certain points remain to be investigated. An excessive sensitivity to inner crowding was not observed in all patients with glaucoma. Central crowding was observed for tiny faces and for a short exposure duration. The mechanisms underlying the greater sensitivity to central crowding in some patients have yet to be understood. Lateral masking, fixation stability, reduced covert attention, contextual modulation might have played a role and remain to be investigated as well as the generalizability of a greater sensitivity to crowding with other stimuli, such as words and objects. 

involves both deep local, and shallow widespread, retinal nerve fiber damage of the macular region. Invest Ophthalmol Vis Sci. 2014; 55:632-649.

344

2.Hood DC. Improving our understanding, and detection, of glaucomatous damage: an approach based upon optical coherence tomography (OCT). Prog Retin Eye Res. 2017;57: 46-75.

3.Nelson P, Aspinall P, Papasouliotis O, Worton B, O’Brien C. Quality of life in glaucoma and its relationship with visual function. J Glaucoma. 2003; 12:139-150.

4.Kwon M, Liu R, Patel BN, Girkin C. Slow reading in glaucoma: is it due to the shrinking visual span in central vision? Invest Ophthalmol Vis Sci. 2017; 58:5810-5818.

5. Glen FC, Crabb DP. Living with glaucoma: a qualitative study of functional implications and patients' coping behaviours. BMC Ophthalmol. 2015; 15:128.

6.Glen FC, Crabb DP, Smith ND, Burton R, \& Garway-Heath DF. Do patients with glaucoma have difficulty recognizing faces? Invest Ophthalmol Vis Sci. 2012;53(7):3629-37.

7. Roux-Sibilon, A., Rutgé, F., Aptel, F., Attye, A., Guyader, N., Boucart, M., Chiquet C Peyrin, C. Scene and human face recognition in the central vision of patients with glaucoma. PLoS One. 2018 Feb 26;13(2): e0193465.

8. Schafer A, Rouland JF, Peyrin C, Szaffarczyk S, Boucart M. Glaucoma affects viewing distance for recognition of sex and facial expression. Invest Ophthalmol Vis Sci. 2018; 59: (12):4921-4928. doi: 10.1167/iovs.18-24875.

9. Mazzoli LS, Urata CN, Kasahara N. Face memory deficits in subjects with eye diseases: a comparative analysis between glaucoma and age-related macular degeneration patients from a developing country. Graefe's Archive for Clin and Exp Ophthalmol. 2019; 257:1941-1946 
10. Glen FC, Smith ND, \& Crabb DP. Saccadic eye movements and face recognition performance in patients with central glaucomatous visual field defects. Vis Res. 2013;82:4251.

11. Manassi M, Whitney D. Multi-level Crowding and the Paradox of Object Recognition in Clutter. Current Biology. 2018; 28: R127-R133 https://doi.org/10.1016/j.cub.2017.12.051

12. Sayim B, Wagemang J. Appearance changes and error characteristics in crowding revealed by drawings. $J$ of Vis. 2017; 17(11):8, 1-16.

13. Martelli M, Majaj NJ, Pelli DG. Are faces processed like words? A diagnostic test for recognition by parts. J of Vis. 2005; 5: 58-70 http://journalofvision.org/5/1/6/ 58

14. Bouma H. Interaction effects in parafoveal letter recognition. Nature. 1970; 226: 177-178 15. Pelli DG, Palomares M, Majaj NJ. Crowding is unlike ordinary masking: Distinguishing feature integration from detection. J of Vis. 2004; 4: 1136-1169

http://journalofvision.org/4/12/12/ 1136

16. Ogata NG, Boer ER, Daga FB, Jammal AA, Stringham JM, Medeiros FA. Visual crowding in glaucoma. Invest Ophthalmol Vis Sci. 2019; 60:538-543.

https://doi.org/10.1167/iovs.18-25150

17. Coates DR, Levi DM, Touch P, Sabesan R. Foveal Crowding Resolved. Scientific Reports. 2018; 8: 9177 | DOI:10.1038/s41598-018-27480-4

18. Sayim B, Westheimer G, Herzog MH. Gestalt factors modulate basic spatial vision. Psychol Sci. 2010; 21: 641-644.

19. Folstein MF, Folstein SE McHugh PR. "Mini-mental state": a practical method for grading the cognitive state of patients for the clinician. J Psychiatric Res. 1975;12: 189-198.

20. Tottenham, N., Tanaka, J.W., Leon, A., McCarry, T., Nurse, M., Hare, T.A. Nelson, C. The NimStim set of facial expressions: Judgments from untrained research participants. Psychiatry Res. 2009;168(3), 242-249 
400

401

402

403

404

405

406

407

408

409

410

411

412

413

414

415

416

417

418

419

420

421

422

423

424

425

426

21. Pluháček F, Siderov J. Mesopic visual acuity is less crowded. Graefes Arch Clin Exp Ophthalmol. 2018 Sep;256(9):1739-1746. doi: 10.1007/s00417-018-4017-6. Epub 2018 May 28

22.Burton R, Smith ND, Crabb DP. Eye movements and reading in glaucoma: observations on patients with advanced visual field loss. Graefes Arch Clin Exp Ophthalmol. 2014; 252:1621-1630

23. Smith ND, Glen FC, M“onter VM, Crabb DP. Using eye tracking to assess reading performance in patients with glaucoma: a within-person study. J Ophthalmol. 2014; 2014: e20528.

24. Lenoble Q, Lek JJ, McKendrick AM. Visual object categorisation in people with glaucoma. Br J Ophthalmol. 2016; 100:1585-1590.

25. Asfaw DS, Jones PR, Mönter VM, Smith ND, Crabb DP. Does glaucoma alter eye movements when viewing images of natural scenes? A between-eye study. Invest Ophthalmol. Vis Sci. 2018; 59:3189-3198

26. Herzog MH, Sayim B, Chicherov V, Manassi M. Crowding, grouping, and object recognition: A matter of appearance. J of Vis. 2015; 15:5, 1-18.

27. Levi DM, Klein SA, Hariharan S. Suppressive and facilitatory spatial interactions in foveal vision: foveal crowding is simple contrast masking. J of Vis. 2002; 2: 140-166

28. Lahav K, Levkovitch-Verbin H, Belkin M, Glovinsky J, Polat U. Reduced Mesopic and Photopic Foveal Contrast Sensitivity in Glaucoma. Arch Ophthalmol. 2011;129 (1): 16-22

29. Ichhpujani P, Thakur S, Spaeth GL. Contrast sensitivity and Glaucoma. J Glaucoma. ( 2020; 29: 71-75

30. Montesano G, Crabb DP, Jones PR, Fogagnolo P, Digiuni M, Rossetti LM.

Evidence for alterations in fixational eye movements in glaucoma. BMC Ophthalmol. 2018; 18:191 https://doi.org/10.1186/s12886-018-0870-7 
427 31. Carrasco M, Penpeci-Talgar C, Eckstein M. Spatial covert attention increases contrast 428 sensitivity along the CSF: Support for signal enhancement. Vis Res. 40; 1203-1215.

429

430 32. Yeshurun Y, Rashal E. Precueing attention to the target location diminishes

431 crowding and reduces the critical distance. J of Vis. 2010; 10:16, 1-12

432

433 33.Sayim B, Westheimer G, Herzog MH. Contrast polarity, chromaticity, and stereoscopic 434 depth modulate contextual interactions in vernier acuity. J of Vis. 2008; 8: 1-9 


\section{LEGENDS}

437

438 Table 1. Characteristics of the two populations (P1-P17 for patients and C1-C17 for controls).

$439 \mathrm{LE}=$ left eye. $\mathrm{RE}=$ right eye. $\mathrm{MD}=$ mean deviation. $\mathrm{RNFL}=$ retinal nerve fiber layer.

440 GCIPL = ganglion cell-inner plexiform layer. Acuity is expressed in LogMar.

441

442 Fig.1. The left and right 10-2 visual fields of the 17 patients.

443

444 Fig.2. Example of face and isolated mouth, closed and open.

445

446 Fig.3. Mean accuracy and standard errors for patients and controls as a function of angular size and crowding condition (isolated mouth versus mouth in face).

448

449 Fig.4. Sensitivity to crowding (accuracy for mouth in face - accuracy for isolated mouth) for 450 each patient at three angular sizes $\left(0.6^{\circ} \mathrm{X} 0.4^{\circ}, 1^{\circ} \times 0.72^{\circ}\right.$ and $\left.1.5^{\circ} \times 1.08^{\circ}\right)$.

451

452

453

454 


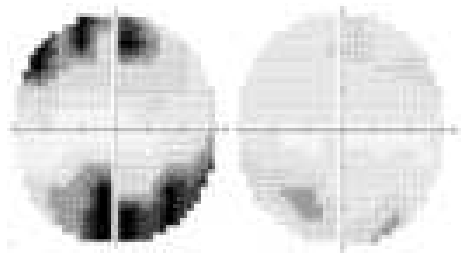

P2

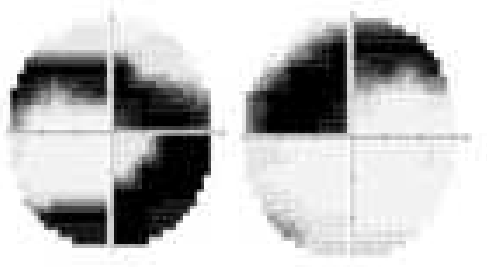

P5
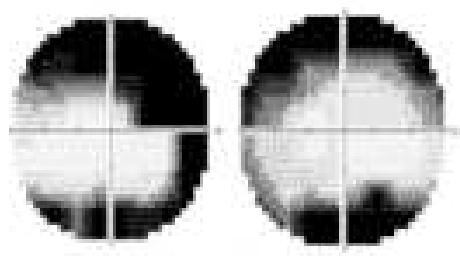

P7

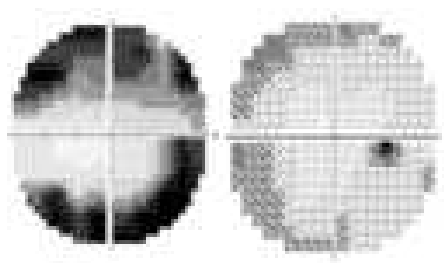

P10

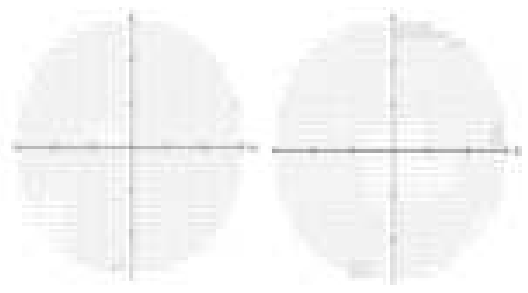

P13

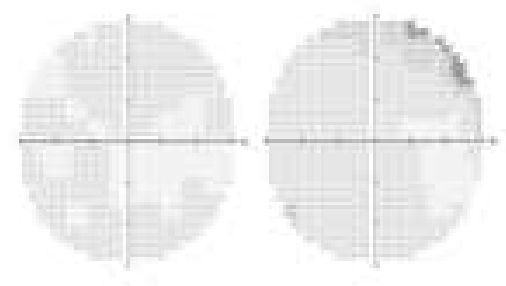

P16

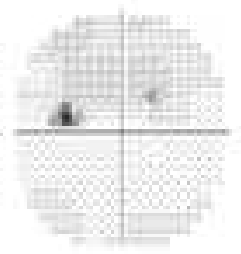

P8

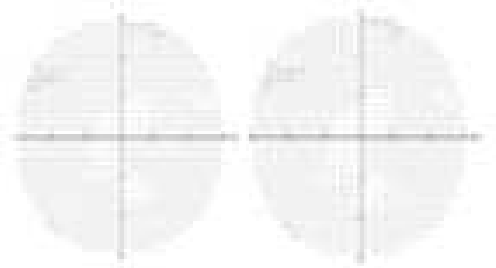

P11

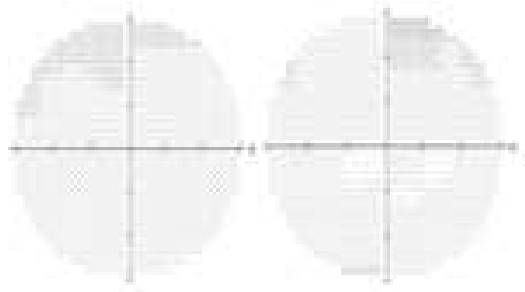

P14

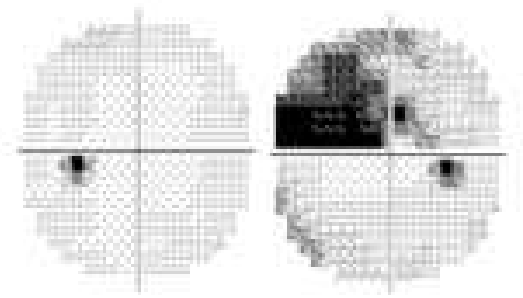

P17

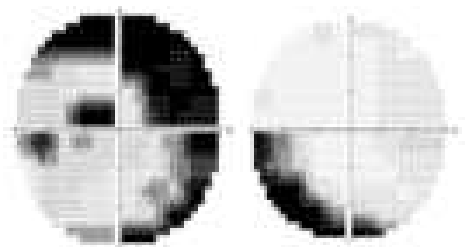

P3

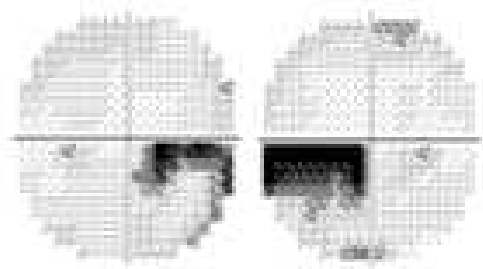

P6

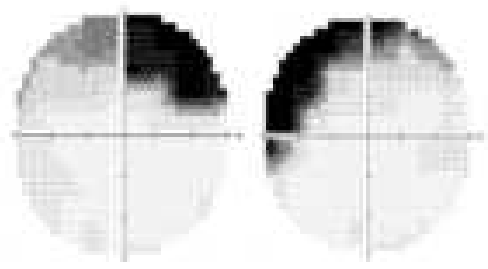

P9

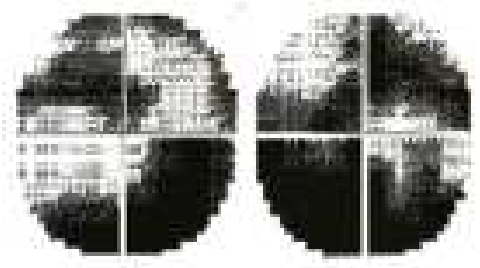

P12

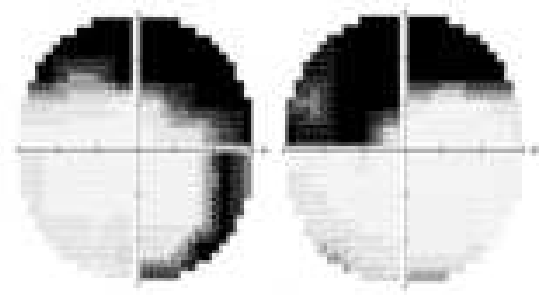

P15 
Figure 2

Click here to access/download;Figure (one figure per file);fig $2 \underline{\underline{ }}$

fig 2 27042020.pdf

glaucoma crowding 1006 2020.tif
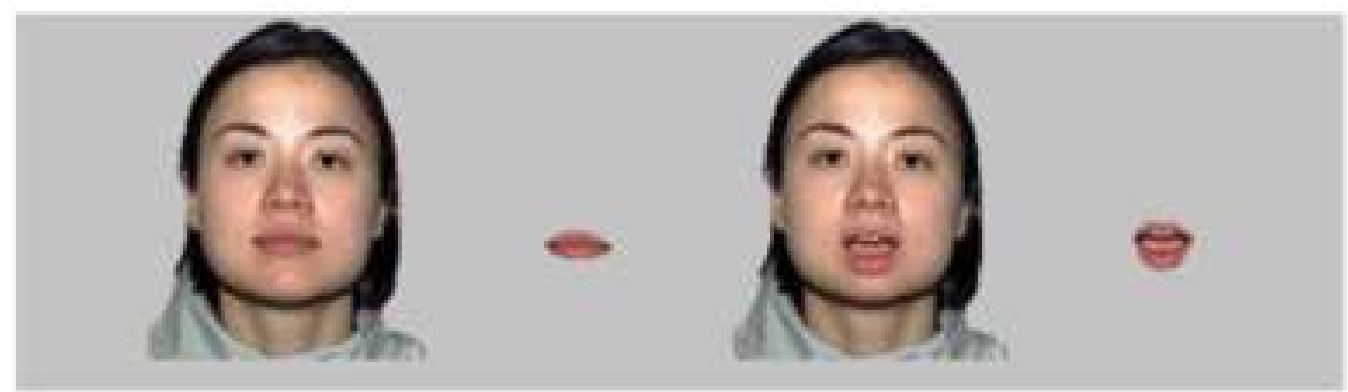
Fig 3 :

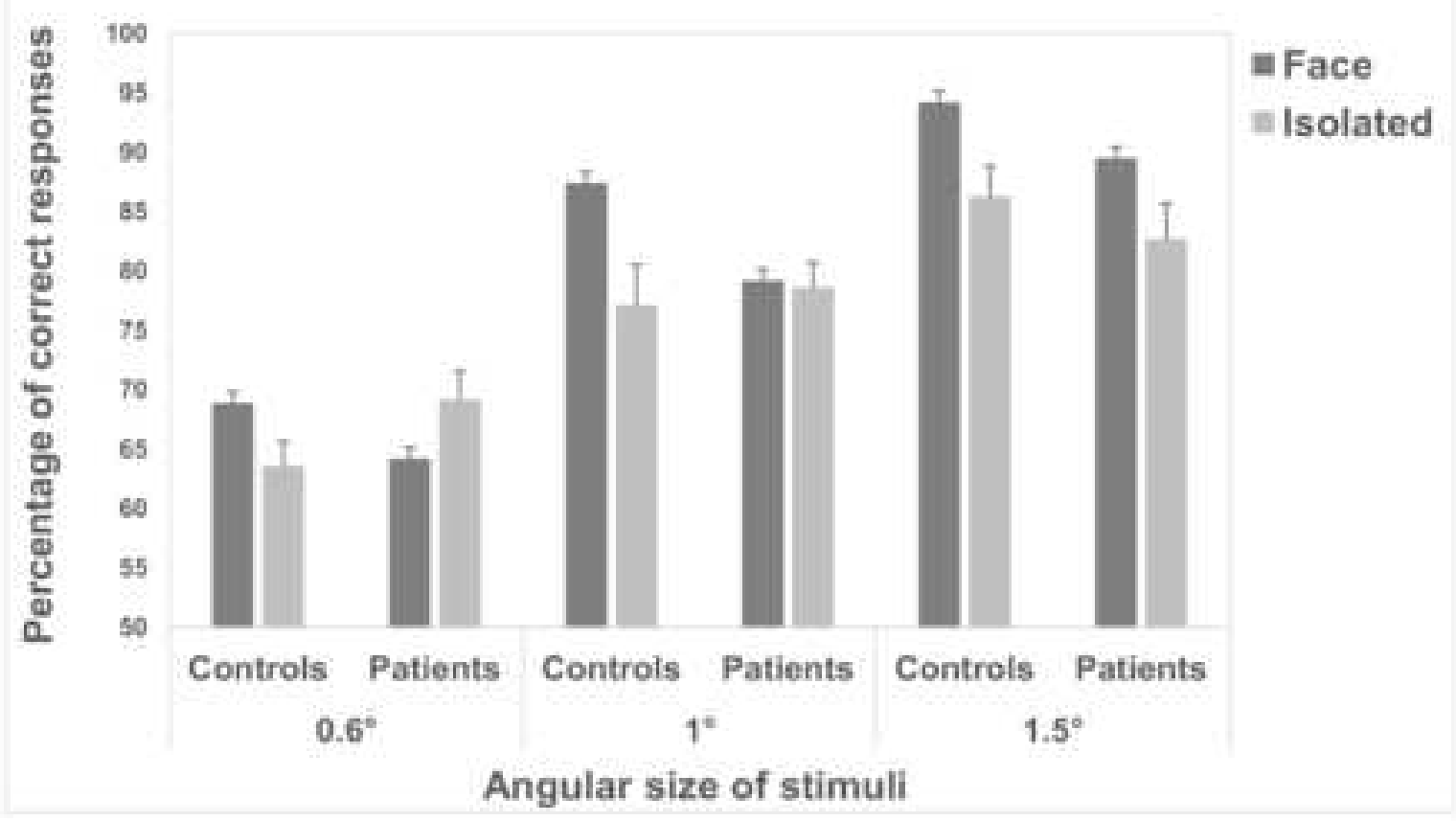


Figure4

fig4 27042020.pdf

ure (one figure per file);fig4 glaucoma crowding10 06 2020.tif

$\mathrm{Fo}_{4}$

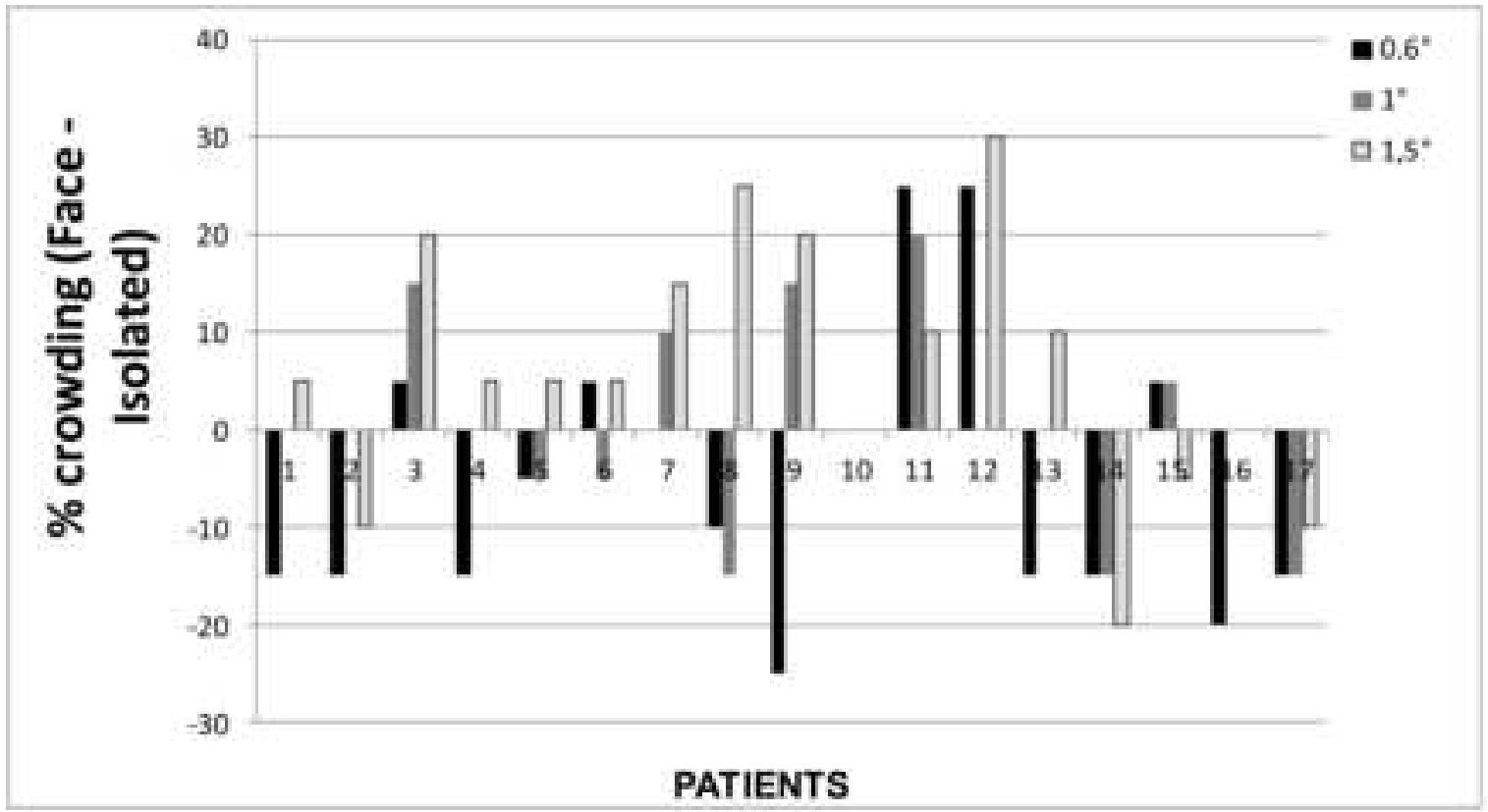




\section{Table1}

\begin{tabular}{ccccccccccc}
\hline Patients & Age & Sex & Contrast & Acuity & MD LE & RNLF LE & GCIPL LE & MD RE & RNLF RE & GCIPL RE \\
\hline P1 & 58 & F & 1.95 & 0 & -5.3 & 72 & 71 & $-10,88$ & 69 & 74 \\
P2 & 49 & M & 1.8 & 0 & -12.88 & 57 & 55 & $-6,17$ & 53 & 54 \\
P3 & 64 & F & 1.5 & 0 & -17.96 & 57 & 52 & $-6,19$ & 71 & 58 \\
P4 & 53 & M & 1.35 & 0.1 & -17.71 & 49 & 49 & $-26,33$ & 56 & 52 \\
P5 & 67 & F & 1.95 & 0 & -17.95 & 64 & 59 & $-10,3$ & 58 & 64 \\
P6 & 58 & M & 1.8 & 0 & -6.33 & 59 & 67 & $-10,15$ & 62 & 64 \\
P7 & 59 & F & 1,95 & 0 & -7.34 & 81 & 61 & $-12,22$ & 82 & 58 \\
P8 & 66 & F & 1.8 & 0 & -19.23 & 55 & 54 & $-10,46$ & 58 & 55 \\
P9 & 70 & F & 1.65 & 0.1 & -7.1 & 76 & 73 & $-7,04$ & 78 & 74 \\
P10 & 68 & F & 1.8 & 0 & -20.86 & 49 & 43 & $-8,55$ & 51 & 43 \\
P11 & 40 & M & 1.8 & 0 & -0.86 & 60 & 64 & $-0,08$ & 73 & 74 \\
P12 & 59 & M & 1.8 & 0 & -18.36 & 46 & 50 & $-23,24$ & 54 & 47 \\
P13 & 59 & F & 1.65 & 0 & -2.01 & 78 & 67 & $-1,71$ & 81 & 68 \\
P14 & 24 & M & 1.95 & 0 & -1.16 & 75 & 73 & $-1,31$ & 81 & 72 \\
P15 & 56 & M & 1.8 & 0 & -13.25 & 44 & 53 & $-11,95$ & 49 & 54 \\
P16 & 69 & F & 1.8 & 0 & -2.03 & 87 & 74 & $-3,47$ & 69 & 67 \\
P17 & 72 & F & 1.65 & 0 & -22.46 & 46 & 51 & $-2,76$ & 75 & 64 \\
\hline
\end{tabular}

\begin{tabular}{ccccc}
\hline Controls & Age & Sex & Contrast & Acuity \\
\hline C1 & 62 & M & 1.8 & 0 \\
C2 & 58 & F & 1.95 & 0.1 \\
C3 & 58 & F & 1.95 & 0 \\
C4 & 56 & F & 1.95 & 0 \\
C5 & 66 & M & 1.8 & 0 \\
C6 & 58 & M & 1.8 & 0 \\
C7 & 64 & F & 1.8 & 0 \\
C8 & 59 & M & 1.95 & 0 \\
C9 & 56 & M & 1.8 & 0 \\
C10 & 56 & M & 1.8 & 0 \\
C11 & 56 & F & 1.8 & 0 \\
C12 & 63 & $\mathrm{~F}$ & 1.65 & 0 \\
C13 & 28 & M & 1.8 & 0 \\
C14 & 59 & F & 1.65 & 0 \\
C15 & 71 & M & 1.8 & 0 \\
C16 & 42 & F & 1.8 & 0 \\
C17 & 73 & M & 1.8 & 0 \\
\hline
\end{tabular}

